\title{
Intermodal Coupling as a Probe for Detecting Nanomechanical Modes
}

\author{
Atakan B. Arı, ${ }^{1}$ M. Çağatay Karakan, ${ }^{1}$ Cenk Yanık, ${ }^{3}$ İsmet İ. Kaya, ${ }^{3,4}$ and M. Selim Hanay, ${ }^{1,2, *}$ \\ ${ }^{1}$ Department of Mechanical Engineering, Bilkent University, 06800 Ankara, Turkey \\ ${ }^{2}$ UNAM-Institute of Materials Science and Nanotechnology, 06800 Ankara, Turkey \\ ${ }^{3}$ SUNUM-Sabancı University Nanotechnology Research and Application Center, 34956 İstanbul, Turkey \\ ${ }^{4}$ Faculty of Engineering and Natural Sciences, Sabanci University, 34956 İstanbul, Turkey
}

(Received 15 August 2017; revised manuscript received 6 December 2017; published 26 March 2018)

\begin{abstract}
Nanoelectromechanical systems provide ultrahigh performance in sensing applications. The sensing performance and functionality can be enhanced by utilizing more than one resonance mode of a nanoelectromechanical-systems device. However, it is often challenging to measure mechanical modes at high frequencies or modes that couple weakly to output transducers. In this paper, we propose the use of intermodal coupling as a mechanism to enable the detection of such modes. To implement this method, a probe mode is continuously driven and monitored using a phase-locked loop, while an auxiliary drive signal scans for other modes. Each time the auxiliary drive signal excites the corresponding mode by matching the mechanical frequency, the effective tension within the structure increases, which in turn causes a frequency shift in the probe mode. The location and width of these frequency shifts can be used to determine the frequency and quality factor of mechanical modes indirectly. Intermodal coupling can be used as a tool to obtain the spectrum of a mechanical structure even if some of these modes cannot be detected conventionally.
\end{abstract}

DOI: 10.1103/PhysRevApplied.9.034024

\section{INTRODUCTION}

Nanomechanical resonators have been used in a wide range of demanding sensor applications such as gas sensing [1-3], single-molecule mass sensing [4], single-protein [5] and neutral-particle [6] mass spectrometry, force sensing [7,8], and inertial imaging [9]. A common trend in recent years has been the exploitation of higher-order modes of a mechanical sensor in mass [5,6,10-15], force [7,8,16], stiffness [13,17], and spatial sensing $[9,18,19]$ where the extra information obtained from the higher-order modes usually expands the types of measurements and increases the sensitivity at the same time [20,21]. Higher-order modes can be utilized for numerous applications, such as quality factor control [22], mechanical vibration registers [23], or phonon cavities [24,25]. However, readout of nanomechanical modes can be challenging under certain conditions. For instance, at higher frequencies, readout schemes tend to get inefficient, e.g., due to increased parasitic capacitive coupling [26]. Moreover, the motion of particular modes may fail to induce a discernible change in the readout transducer; for instance, the readout signal for even-parity modes is canceled out in magnetomotive [27] and optomechanical [28] detection techniques.

In this article, we describe a comprehensive approach to obtain the frequency spectrum of micromechanical and nanomechanical resonators. We use the observation that the

\footnotetext{
*selimhanay@bilkent.edu.tr
}

flexural modes of a doubly clamped beam couple to each other anharmonically through the increase of tension on the beam [29-32]. As a doubly clamped beam undergoes flexural motion, the increased tension on the beam tunes the frequency of the other modes. This coupling mechanism is used to detect modes indirectly in earlier experiments at the MEMS scale using two-dimensional frequency-frequency sweeps [30] where one sweep for the probe and another sweep for the pump mode is utilized. Instead of using two concatenated frequency sweeps (which would require a 2D search space), here we keep one mode at resonance using a phaselocked-loop (PLL) circuit and sweep for the other mode only. This way the search space decreases to $1 \mathrm{D}$, which increases the speed of detection. Moreover, our experiments are performed at the nanoelectromechanical-systems (NEMS) scale and demonstrate modes as high as $840 \mathrm{MHz}$, which is at several orders of magnitude higher frequency than previous top-down device demonstrations. Finally, we incorporate a two-tier verification system using two different probe modes for eliminating any unintentional drive of different modes.

The proposed technique is realized by tracking the resonance frequency of a lower-order mode (e.g., the first or the second mode) of a resonator using a PLL, while performing a frequency sweep in parallel to excite the other modes using the same actuation electrode [29]. This simultaneous combination of a frequency sweep and PLL allows for the efficient detection of higher modes that can be driven but cannot be detected with conventional methods. For the transduction of the device we use thermoelastic actuation 
and piezoresistive detection in our measurements since this combination offers an efficient platform to investigate higher-order modes [33]. However, the proposed technique can be used with any transduction scheme as long as there is a mechanical coupling mechanism between the modes.

\section{DEVICE FABRICATION}

Fabrication of the device [Fig. 1(a)] starts with a 100-nm-thick silicon nitride on a silicon substrate. Contact pads and electrodes are patterned using electronbeam lithography followed by deposition of a 100-nmthick gold layer with a 4-nm chromium adhesion layer underneath. The beam layout is then patterned using electron-beam lithography, and a 60-nm-thick copper layer is deposited to be used as an etch mask. Inductively coupled plasma is used for dry etching: silicon nitride is etched anisotropically followed by the isotropic release of the doubly clamped beam. After removal of the mask layer, the device is wire bonded to a printed circuit board for electrical and mechanical characterization. The final structure is $20 \mu \mathrm{m}$ long, $320 \mathrm{~nm}$ wide, and $100 \mathrm{~nm}$ thick [Fig. 1(a)]. The gold electrodes are $780 \mathrm{~nm}$ long and $80 \mathrm{~nm}$

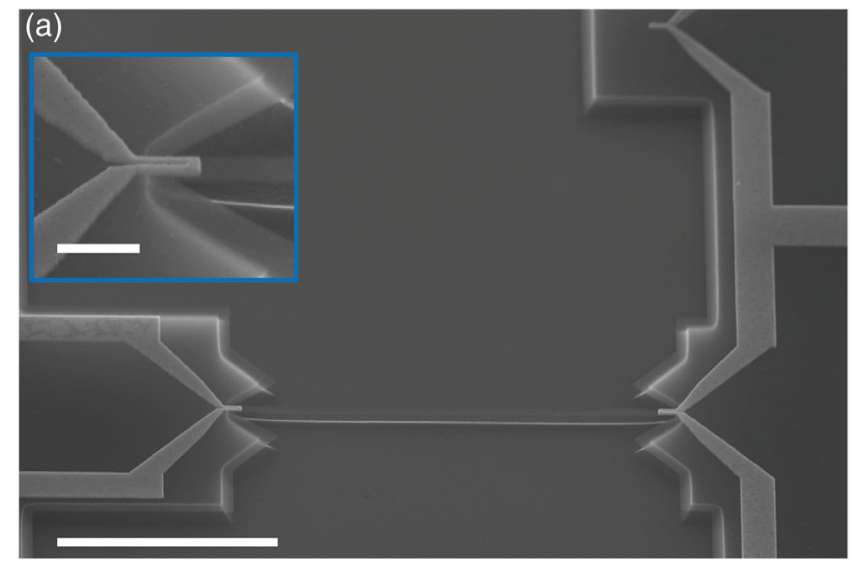

(b)

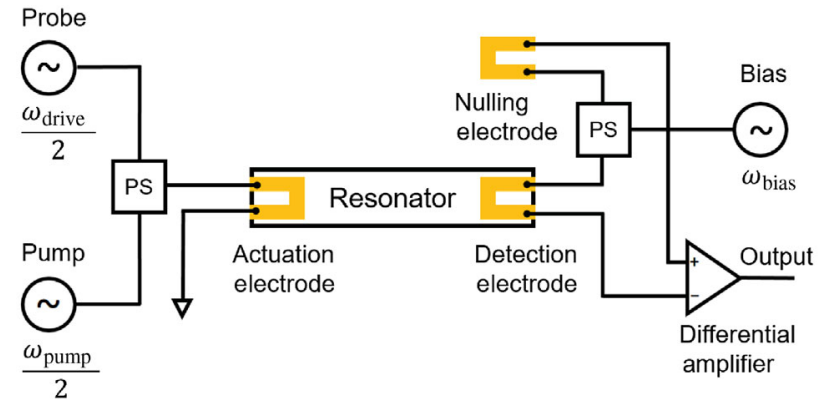

FIG. 1. (a) Scanning electron micrograph of a nanomechanical resonator along with the electrodes for electrothermal actuation, piezoresistive detection, and nulling electrode for background cancellation. Dimensions are $20 \mu \mathrm{m} \times 320 \mathrm{~nm} \times 100 \mathrm{~nm}$, the scale bar is $10 \mu \mathrm{m}$. The inset shows a close-up view of the drive electrode; the scale bar is $1 \mu \mathrm{m}$. (b) Actuation and readout scheme to pump and detect higher-order modes while simultaneously tracking a specific resonance mode with PLL. wide with each leg $80 \mathrm{~nm}$ apart [Fig. 1(a), inset]. Resistances of the electrodes are typically around $35 \Omega$ for actuation and $25 \Omega$ for the detection loop.

\section{EXPERIMENTAL METHOD}

The actuation scheme [33] relies on the difference in the thermal expansion coefficients of the gold electrode and silicon nitride beam. An ac drive voltage $\left(V_{d}\right)$ at half of the mechanical drive frequency $\left(\omega_{d} / 2\right)$ is applied to the drive loop to create local Joule heating [34] [Fig. 1(b)]. The differential expansion of the materials induces stress on the structure and causes elastic deformation at twice the applied frequency. The resulting flexural motion of the beam distorts the detection loop at the other end of the beam, which alters the resistance of the readout electrode $\Delta R(\omega)$ through the geometric piezoresistance effect [35]. To measure the dynamical resistance and eliminate the effect of parasitic capacitances, we use a down-mixing scheme by applying an ac bias voltage $\left(V_{b}\right)$ to the detection loop at a slightly detuned $(<100 \mathrm{kHz})$ frequency of $\omega_{d}+\Delta \omega$. In this way, a clear readout signal at the mixed-down frequency of $\Delta \omega$ emerges. The detection scheme is further enhanced by incorporating a differential readout circuit, utilizing a nulling electrode, anchored to the substrate, to cancel out electronic background.

For the initial characterization and calibration of the nanomechanical modes, frequency sweeps are conducted at 760 Torr (Fig. 2, main panel). Both out-of-plane and inplane modes of the device are detected in these measurements. For each mode, finer frequency response curves are obtained by decreasing the pressure down to $2 \times 10^{-4}$ mTorr and performing narrower frequency scans (Fig. 2, lower insets). Measured resonance frequency values are used in the beam model and an initial longitudinal stress of $860 \mathrm{MPa}$ is determined (Supplemental Material [36]).

Following the open-loop characterization, we want to implement the proposed technique by coupling the modes that have already been detected. For this implementation, the fundamental mode of the device is driven continuously using a PLL circuit. While the PLL tracking is maintained, higher modes of the device are excited with a simultaneous frequency sweep by superposing a pump signal at frequency $\omega_{p}$ on the actuation electrode [Fig. 1(b)]. When the pump signal excites a higher mode of the device, an upward frequency shift is observed in the PLL mode owing to increased tension, which indicates the existence of a mechanical mode (Fig. 2, upper insets). These observations indicate that the location and width of mechanical resonances can be detected through mode coupling.

\section{THEORY}

Coupling between flexural modes occurs due to the increased tension along the beam axis which changes the effective stiffness of the mechanical structure [29]. When a 


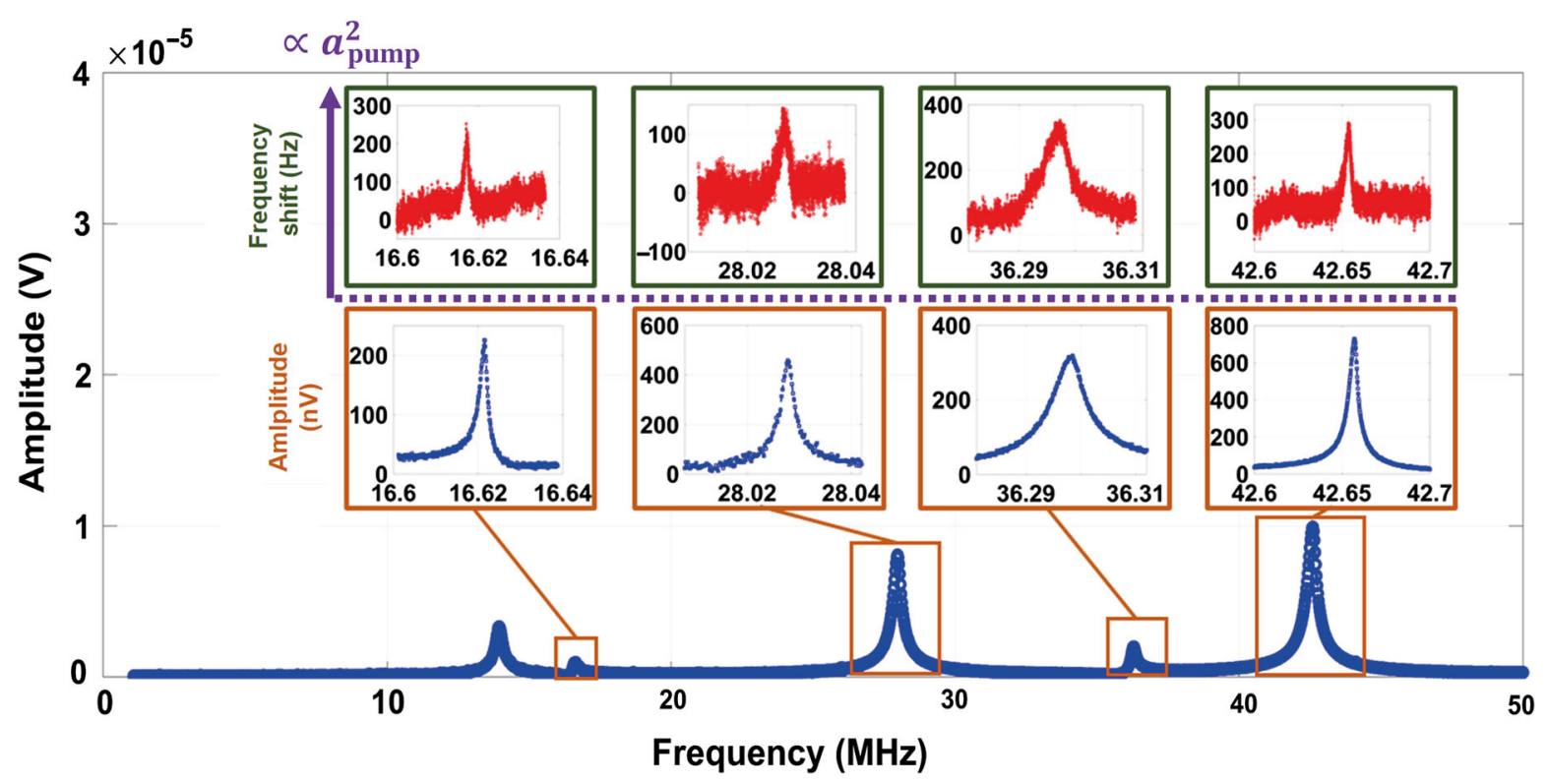

FIG. 2. Open-loop measurement from 1 to $50 \mathrm{MHz}$ in air. In this frequency range, five modes are evident: three out-of-plane modes (which have relatively higher amplitudes) and two in-plane modes. The bottom insets demonstrate linear responses of modes 2-5 at $2 \times 10^{-4}$ Torr with drive voltages of $90,42,211$, and $63 \mathrm{mVpp}$, respectively. The top insets demonstrate frequency shifts induced on the first mode (probe), caused by the intermodal coupling to the pump modes. These shifts are proportional to the square of the pump drive voltage. The applied voltages for the pump are same as the open-loop sweeps and applied voltage to drive the first mode is $32 \mathrm{mVpp}$.

device is actuated, a doubly clamped beam undergoes a flexural motion; however, the total length of the beam is restricted because both ends are clamped, and consequently, the longitudinal tension on the beam increases. The additional tension increases the effective stiffness of the beam, which in turn modulates the frequency of the vibrational modes. As a result, when a mode is excited, the frequency of every other mode gets tuned, depending on its coupling strength to the excited mode. This can be followed by considering the equation of motion for the beam [37]:

$E I \frac{\partial^{4} w}{\partial x^{4}}+\rho A \frac{\partial^{2} w}{\partial t^{2}}=\left[T_{0}+\frac{E A}{2 L} \int_{0}^{L}\left(\frac{\partial w}{\partial x}\right)^{2} d x\right] \frac{\partial^{2} w}{\partial x^{2}}$,

where $E$ is the Young's modulus, $I$ is the moment of inertia, $w=w(x, t)$ is the displacement field, $\rho$ is the density, $A$ is the cross-sectional area, $L$ is the length of the beam. The intrinsic tension on the beam is shown by $T_{0}$. On the righthand side, the term $(\partial w / \partial x)^{2}$ accounts for the length change due to the oscillation of the beam. In general, the displacement field can be decomposed into different modes: $w(x, t)=\sum_{n} a_{n} \phi_{n}(x) \zeta_{n}(t)$.

In the experiments, at most two modes are excited at a given time, therefore, the frequency shift in the measured probe mode $(k)$ due to the excitation of a pump mode $(j)$ can be calculated as $[29,30]$ (see Supplemental Material [36]):

$$
\Delta \omega=\omega_{k}-\omega_{k, \text { init }}=\omega_{k, \text { init }} g_{k j} a_{j}^{2},
$$

where $g_{k j}$ is the coupling coefficient between modes $j$ and $k$. As the oscillation amplitude of mode $a_{j}$ starts from zero and increases to large values, the frequency shift observed in

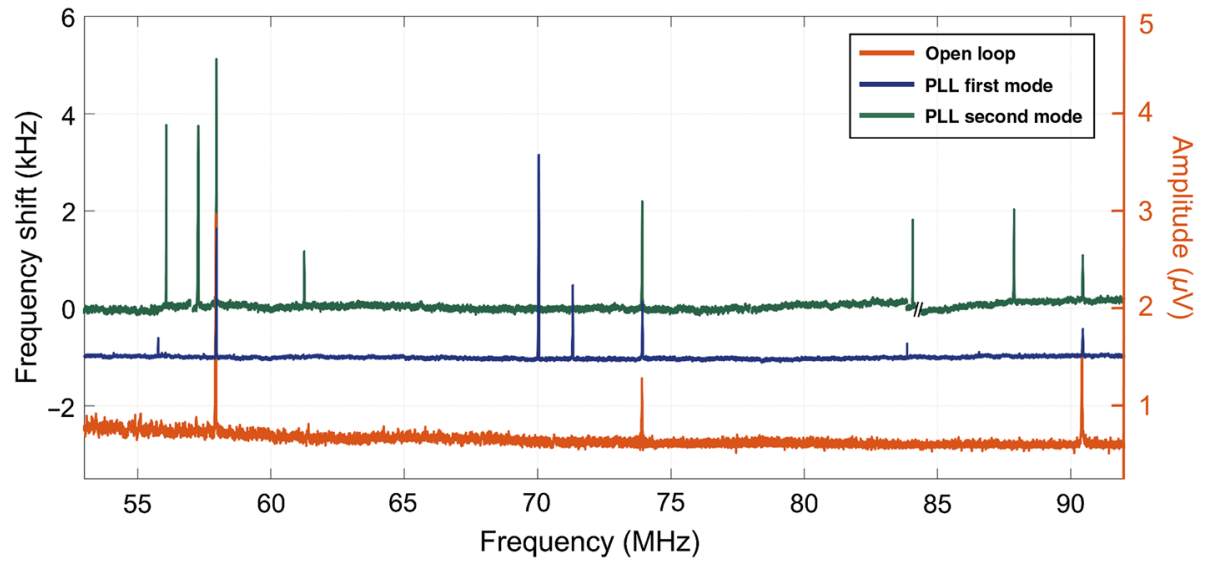

FIG. 3. Overlay of the open-loop response of the device (orange) and the spectrum obtained through the combination of the first mode (blue) and second mode (green) PLLs. In all three cases, the pump signal for exciting modes is $211 \mathrm{mVpp}$. The peaks that overlap on both PLL runs indicate a mechanical mode, whereas other peaks are due to extra driving terms caused by the thermoelastic actuation. 
the probe mode $(k)$ can be related to the amplitude of the pump mode [29]: $\Delta \omega_{k} \sim g_{j k} x_{j}^{2}$. In this way, the location of the frequency shift is used to indicate the pump-mode frequency and the magnitude of the frequency shift is used to indicate the square of amplitude.

Next, the detection of mechanical modes at higher frequencies is attempted. This attempt brings about a complication: with thermoelastic actuation used in the experiments, the combination of two ac signals (PLL drive and the pump signal) on the same drive electrode generates additional harmonics, which may excite modes at unintended frequencies. To eliminate such occurrences, a second run is performed by tracking the second out-ofplane mode - instead of the fundamental mode - with PLL and repeating the frequency sweep with the pump signal. Only the frequencies at which both PLL runs have overlapping resonance peaks indicate the existence of a mechanical resonance. To demonstrate the utility of the modified technique, the resonance frequencies of the first two out-of-plane modes are sequentially tracked by a PLL circuitry (Fig. 3). An open-loop sweep is also conducted for independent characterization. As expected, the results show that mechanical modes exist only at locations at which both of the PLL runs have frequency shifts.

\section{RESULTS AND DISCUSSION}

The frequency shifts at the resonance frequencies are in good agreement with the open-loop resonance values of these modes. The quality factors of the modes are also calculated from the frequency shift response of the modes, which agree with the open-loop measurements of the first five modes of the device (Table I). Using the intermodal coupling and detection method, we are able to detect modes up to $840 \mathrm{MHz}$ (Fig. 4), whereas our measurement setup

TABLE I. Quality factors of the first five modes and two ultrahigh-frequency modes are listed. The comparison of quality factors in open-loop and closed-loop (intermodal) measurements are provided. Quality factors are deduced by measuring the full width at half maximum of the power for open-loop measurements. For closed-loop measurements, where the frequency shift signal scales with the square of the amplitude, full width at half maximum of the frequency shift signal is used. The uncertainty in measuring the quality factor is estimated to be no more than $10 \%$ for the worst case.

\begin{tabular}{lcccc}
\hline \hline $\begin{array}{l}\text { Mode } \\
\text { number }\end{array}$ & $\begin{array}{c}\text { Frequency } \\
(\mathrm{MHz})\end{array}$ & $\begin{array}{c}\text { Open-loop } \\
\text { quality factor }\end{array}$ & $\begin{array}{c}\text { Closed-loop } \\
\text { quality factor }\end{array}$ & $\begin{array}{c}\text { Error } \\
(\%)\end{array}$ \\
\hline 1 & 13.65 & 17480 & & \\
2 & 16.62 & 11080 & 11078 & 0.02 \\
3 & 28.03 & 11211 & 11210 & 0.009 \\
4 & 36.3 & 4270 & 4537 & 6.25 \\
5 & 42.67 & 9920 & 10663 & 7 \\
$\ldots$ & 473 & $\ldots$ & 844 & $\ldots$ \\
$\ldots$ & 840 & $\ldots$ & 280 & $\cdots$ \\
\hline \hline
\end{tabular}

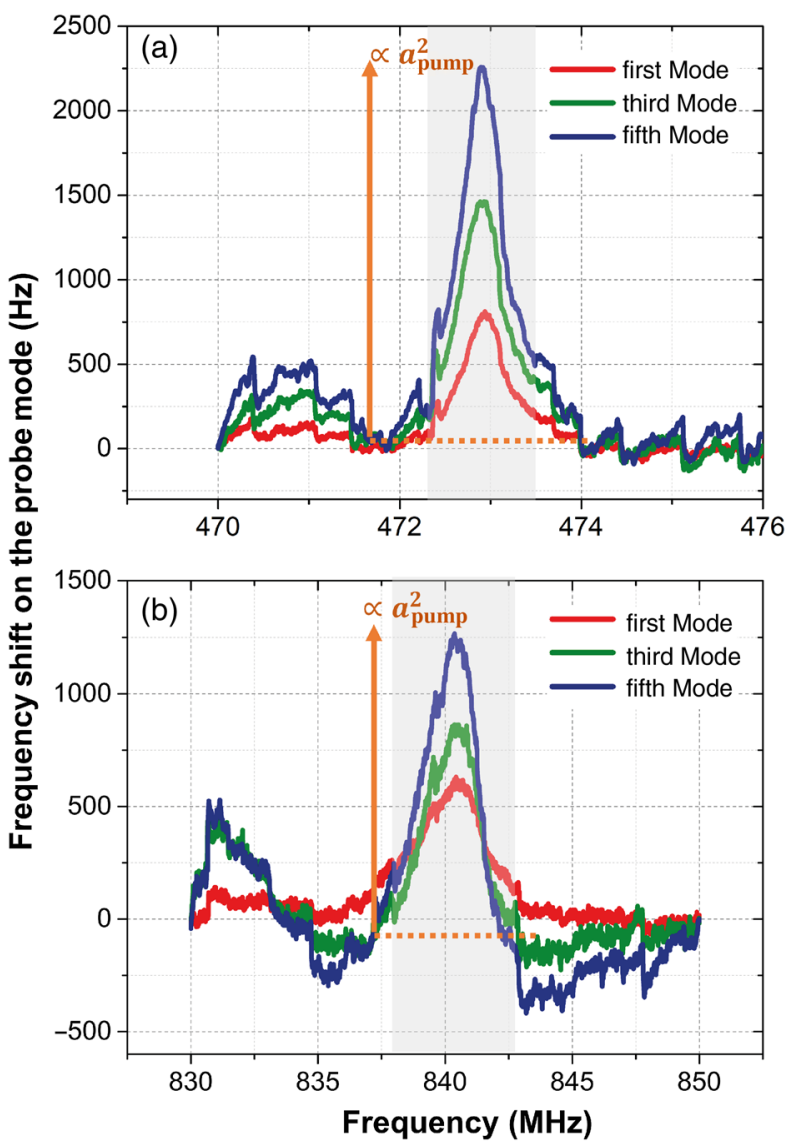

FIG. 4. Intermodal coupling measurements using the first three out-of-plane modes. Two modes are detected at approximately (a) $473 \mathrm{MHz}$ and (b) $840 \mathrm{MHz}$, at which all three probe modes experience significant frequency tuning. For both cases applied pump voltage is $270 \mathrm{mVpp}$ and drive voltages for PLL are 32, 42, and $63 \mathrm{mVpp}$ for the first, third, and fifth modes, respectively (first, second, and third out-of-plane modes). The auxiliary axes inside the charts indicate that these frequency shifts relate to the square amplitude of the pumped (inferred) modes.

for the piezoresistive readout is limited to the $(0-250)-\mathrm{MHz}$ range. Since there are numerous modes that can be detected, we limit our presentation only to those that result in large mode coupling [29] with the first three out-ofplane modes.

The technique proposed here keeps the probe mode at resonance throughout the experiment using a PLL circuit. In this way, the indirect detection of the higher-order modes can be accomplished by performing a frequency sweep only for the pump mode. If $N$ points are used in these frequency sweeps with $\Delta t$ being the duration between data points, the total duration of the technique proposed here scales with $2 N \Delta t$ where the factor 2 comes from the fact that we use a second mode for verification. On the other hand, alternative implementations which use open-loop characterization (instead of PLL) for the probe mode take a longer time: if $M$ points are used for the probe-mode sweep and $N$ points are used for the pump-mode sweep, then the 
total duration for such intermodal detections scales with $M N \Delta t$, which can be orders of magnitude slower since to measure a resonance with reasonable span and resolution, one usually needs at least $M>100$. Therefore, the technique proposed here speeds up the indirect detection of modes through intermodal coupling.

\section{CONCLUSION}

Intermodal coupling offers an alternative method for detecting higher vibrational modes of NEMS, which can be driven relatively easily but cannot be detected efficiently due to drawbacks in the detection method or other instrumental limitations. Indirect detection through intermodal coupling can be implemented effectively by keeping one mode continuously at resonance using a PLL and scanning for other modes. The technique provides a thorough spectrum of nanomechanical modes which can be useful for modeling system parameters accurately and accomplishing multimodal sensing. The method is not limited by the choice of the transduction method and can be applied to any conventional NEMS detection schemes to improve the readout strength of high frequency modes.

\section{ACKNOWLEDGMENTS}

This work was supported by the Scientific and Technological Research Council of Turkey (TÜBİTAK), Grant No. EEEAG-113E600. The authors thank John E. Sader, Matt Matheny, and Kamil L. Ekinci for useful discussions.

[1] M. Li, E. B. Myers, H. X. Tang, S. J. Aldridge, H. C. McCaig, J. J. Whiting, R. J. Simonson, N. S. Lewis, and M. L. Roukes, Nanoelectromechanical resonator arrays for ultrafast, gas-phase chromatographic chemical analysis, Nano Lett. 10, 3899 (2010).

[2] L. L. Li, E. L. Holthoff, L. A. Shaw, C. B. Burgner, and K. L. Turner, Noise squeezing controlled parametric bifurcation tracking of MIP-coated microbeam MEMS sensor for TNT explosive gas sensing, J. Microelectromech. Syst. 23, 1228 (2014).

[3] W. J. Venstra, M. J. Capener, and S. R. Elliott, Nanomechanical gas sensing with nonlinear resonant cantilevers, Nanotechnology 25, 425501 (2014).

[4] A. K. Naik, M. S. Hanay, W. K. Hiebert, X. L. Feng, and M.L. Roukes, Towards single-molecule nanomechanical mass spectrometry, Nat. Nanotechnol. 4, 445 (2009).

[5] M. S. Hanay, S. Kelber, A. K. Naik, D. Chi, S. Hentz, E. C. Bullard, E. Colinet, L. Duraffourg, and M. L. Roukes, Single-protein nanomechanical mass spectrometry in real time, Nat. Nanotechnol. 7, 602 (2012).

[6] E. Sage, A. Brenac, T. Alava, R. Morel, C. Dupré, M. S. Hanay, M. L. Roukes, L. Duraffourg, C. Masselon, and S. Hentz, Neutral particle mass spectrometry with nanomechanical systems, Nat. Commun. 6, 6482 (2015).
[7] E. T. Herruzo, A. P. Perrino, and R. Garcia, Fast nanomechanical spectroscopy of soft matter, Nat. Commun. 5, 3126 (2014).

[8] L. M. de Lépinay, B. Pigeau, B. Besga, P. Vincent, P. Poncharal, and $\mathrm{O}$. Arcizet, A universal and ultrasensitive vectorial nanomechanical sensor for imaging $2 \mathrm{D}$ force fields, Nat. Nanotechnol. 12, 156 (2017).

[9] M. S. Hanay, S. I. Kelber, C. D. O’Connell, P. Mulvaney, J. E. Sader, and M. L. Roukes, Inertial imaging with nanomechanical systems, Nat. Nanotechnol. 10, 339 (2015).

[10] S. Dohn, W. Svendsen, A. Boisen, and O. Hansen, Mass and position determination of attached particles on cantilever based mass sensors, Rev. Sci. Instrum. 78, 103303 (2007).

[11] S. Dohn, S. Schmid, F. Amiot, and A. Boisen, Position and mass determination of multiple particles using cantilever based mass sensors, Appl. Phys. Lett. 97, 044103 (2010).

[12] S. Schmid, S. Dohn, and A. Boisen, Real-time particle mass spectrometry based on resonant micro strings, Sensors $\mathbf{1 0}$, 8092 (2010).

[13] E. Gil-Santos, D. Ramos, J. Martinez, M. FernandezRegulez, R. Garcia, A. San Paulo, M. Calleja, and J. Tamayo, Nanomechanical mass sensing and stiffness spectrometry based on two-dimensional vibrations of resonant nanowires, Nat. Nanotechnol. 5, 641 (2010).

[14] S. Olcum, N. Cermak, S. C. Wasserman, and S. R. Manalis, High-speed multiple-mode mass-sensing resolves dynamic nanoscale mass distributions, Nat. Commun. 6, 7070 (2015).

[15] J. E. Sader, M. S. Hanay, A. P. Neumann, and M. L. Roukes, Mass spectrometry using nanomechanical systems: beyond the point-mass approximation, Nano Lett. 18, 1608 (2018).

[16] R. Garcia and E. T. Herruzo, The emergence of multifrequency force microscopy, Nat. Nanotechnol. 7, 217 (2012).

[17] J. Ruz, J. Tamayo, V. Pini, P. M. Kosaka, and M. Calleja, Physics of nanomechanical spectrometry of viruses, Sci. Rep. 46015 (2014).

[18] T. Biswas, J. Xu, N. Miriyala, C. Doolin, T. Thundat, J. Davis, and K. Beach, Time-Resolved Mass Sensing of a Molecular Adsorbate Nonuniformly Distributed Along a Nanomechnical String, Phys. Rev. Applied 3, 064002 (2015).

[19] S. M. Heinrich and I. Dufour, Toward higher-order mass detection: Influence of an adsorbate's rotational inertia and eccentricity on the resonant response of a Bernoulli-Euler cantilever beam, Sensors 15, 29209 (2015).

[20] M. K. Ghatkesar, V. Barwich, T. Braun, J.-P. Ramseyer, C. Gerber, M. Hegner, H. P. Lang, U. Drechsler, and M. Despont, Higher modes of vibration increase mass sensitivity in nanomechanical microcantilevers, Nanotechnology 18, 445502 (2007).

[21] D. Ramos, J. Tamayo, J. Mertens, M. Calleja, L. Villanueva, and A. Zaballos, Detection of bacteria based on the thermomechanical noise of a nanomechanical resonator: origin of the response and detection limits, Nanotechnology 19, 035503 (2008).

[22] W. J. Venstra, H. J. Westra, and H. S. van der Zant, $Q$-factor control of a microcantilever by mechanical sideband excitation, Appl. Phys. Lett. 99, 151904 (2011).

[23] I. Mahboob, V. Nier, K. Nishiguchi, A. Fujiwara, and H. Yamaguchi, Multi-mode parametric coupling in an electromechanical resonator, Appl. Phys. Lett. 103, 153105 (2013). 
[24] I. Mahboob, K. Nishiguchi, H. Okamoto, and H. Yamaguchi, Phonon-cavity electromechanics, Nat. Phys. 8, 387 (2012).

[25] R. De Alba, F. Massel, I. Storch, T. Abhilash, A. Hui, P. McEuen, H. Craighead, and J. Parpia, Tunable phononcavity coupling in graphene membranes, Nat. Nanotechnol. 11, 741 (2016).

[26] I. Bargatin, E. Myers, J. Arlett, B. Gudlewski, and M. Roukes, Sensitive detection of nanomechanical motion using piezoresistive signal downmixing, Appl. Phys. Lett. 86, 133109 (2005).

[27] A. Cleland and M. Roukes, Fabrication of high frequency nanometer scale mechanical resonators from bulk Si crystals, Appl. Phys. Lett. 69, 2653 (1996).

[28] J. Westwood-Bachman, Z. Diao, V. Sauer, D. Bachman, and W. Hiebert, Even nanomechanical modes transduced by integrated photonics, Appl. Phys. Lett. 108, 061103 (2016).

[29] M. Matheny, L. Villanueva, R. Karabalin, J. E. Sader, and M. Roukes, Nonlinear mode-coupling in nanomechanical systems, Nano Lett. 13, 1622 (2013).

[30] H. Westra, M. Poot, H. Van Der Zant, and W. Venstra, Nonlinear modal interactions in clamped-clamped mechanical resonators, Phys. Rev. Lett. 105, 117205 (2010).
[31] A. Eichler, M. del Álamo Ruiz, J. Plaza, and A. Bachtold, Strong coupling between mechanical modes in a nanotube resonator, Phys. Rev. Lett. 109, 025503 (2012).

[32] P. Truitt, J. Hertzberg, E. Altunkaya, and K. Schwab, Linear and nonlinear coupling between transverse modes of a nanomechanical resonator, J. Appl. Phys. 114, 114307 (2013).

[33] I. Bargatin, I. Kozinsky, and M. Roukes, Efficient electrothermal actuation of multiple modes of high-frequency nanoelectromechanical resonators, Appl. Phys. Lett. 90, 093116 (2007).

[34] L. Jiang, R. Cheung, J. Hedley, M. Hassan, A. Harris, J. Burdess, M. Mehregany, and C. Zorman, SiC cantilever resonators with electrothermal actuation, Sens. Actuators A 128, 376 (2006).

[35] M. Li, H. X. Tang, and M. L. Roukes, Ultra-sensitive NEMS-based cantilevers for sensing, scanned probe and very high-frequency applications, Nat. Nanotechnol. 2, 114 (2007).

[36] See Supplemental Material at http://link.aps.org/ supplemental/10.1103/PhysRevApplied.9.034024 for the details of calculations and experimental implementation.

[37] E. M. Abdel-Rahman, M. I. Younis, and A. H. Nayfeh, Characterization of the mechanical behavior of an electrically actuated microbeam, J. Micromech. Microeng. 12, 759 (2002). 